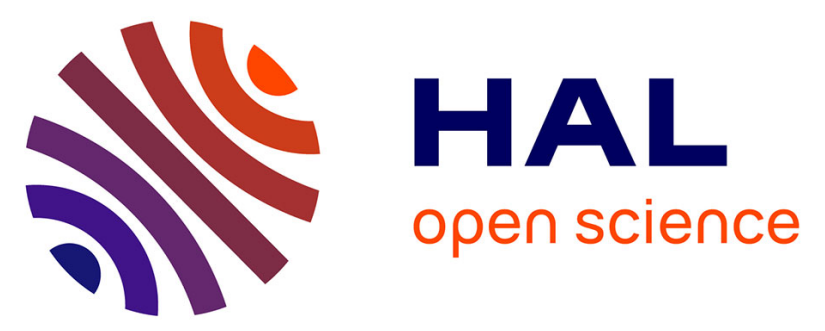

\title{
Recovery of surface state bands after desorption of Te capping layer on (Bi1-xSbx)2Te3 ternary topological insulators
}

Geoffroy Kremer, Kejing Zhu, Thomas Pierron, Vincent Fournee, Julian Ledieu, Stéphane Andrieu, Bertrand Kierren, Luc Moreau, Daniel Malterre, Ke He, et al.

\section{To cite this version:}

Geoffroy Kremer, Kejing Zhu, Thomas Pierron, Vincent Fournee, Julian Ledieu, et al.. Recovery of surface state bands after desorption of Te capping layer on (Bi1-xSbx)2Te3 ternary topological insulators. Journal of Physics D: Applied Physics, 2019, 52 (49), pp.494002. 10.1088/1361-6463/ab3fc3 . hal-02999617

\section{HAL Id: hal-02999617 https://hal.science/hal-02999617}

Submitted on 20 Nov 2020

HAL is a multi-disciplinary open access archive for the deposit and dissemination of scientific research documents, whether they are published or not. The documents may come from teaching and research institutions in France or abroad, or from public or private research centers.
L'archive ouverte pluridisciplinaire HAL, est destinée au dépôt et à la diffusion de documents scientifiques de niveau recherche, publiés ou non, émanant des établissements d'enseignement et de recherche français ou étrangers, des laboratoires publics ou privés. 


\title{
Recovery of surface state bands after desorption of Te capping layer on $\left(\mathrm{Bi}_{1-\mathrm{x}} \mathrm{Sb}_{\mathrm{x}}\right)_{2} \mathrm{Te}_{3}$ ternary topological insulators
}

Geoffroy Kremer, ${ }^{1}$ Kejing Zhu, ${ }^{2}$ Thomas Pierron, ${ }^{1}$ Vincent Fournee, ${ }^{1}$ Julian Ledieu, ${ }^{1}$ Stéphane Andrieu, ${ }^{1}$ Bertrand Kierren, ${ }^{1}$ Luc Moreau, ${ }^{1}$ Daniel Malterre, ${ }^{1} \mathrm{Ke} \mathrm{He},{ }^{2}$ Qi-Kun Xue, ${ }^{2}$ Yannick Fagot-Revurat ${ }^{1 *}$ and Yuan $\mathrm{Lu}^{1 \dagger}$

${ }^{1}$ Université de Lorraine, CNRS, Institut Jean Lamour, UMR 7198, campus ARTEM, 2 Allée André Guinier, 54011 Nancy, France

${ }^{2}$ State Key Laboratory of Low Dimensional Quantum Physics, Department of Physics, Tsinghua University, Beijing 100084, P. R. China

Corresponding authors:

*yannick.fagot@univ-lorraine.fr; †yuan.lu@univ-lorraine.fr

\begin{abstract}
Te capping layer on topological insulator (TI) surface provides an efficient way for the protection of surface from oxidation. However, when using Te to protect the surface of $\left(\mathrm{Bi}_{1-\mathrm{x}} \mathrm{Sb}_{\mathrm{x}}\right)_{2} \mathrm{Te}_{3}$ ternary topological insulators, it is still unclear for the influence of Te on the surface chemical composition and the surface state bands after the procedure of capping and desorption of Te. Here, we have performed a systematic study of the surface morphology, crystallinity, chemical composition as well as the band structure of $\left(\mathrm{Bi}_{1-\mathrm{x}} \mathrm{Sb}_{\mathrm{x}}\right)_{2} \mathrm{Te}_{3}$ after the desorption of the Te capping layer. Our results confirm a good recovery of the surface state bands of the $\left(\mathrm{Bi}_{1-}\right.$ $\left.{ }_{x} \mathrm{Sb}_{\mathrm{x}}\right)_{2} \mathrm{Te}_{3}$ ternary topological insulators with different $\mathrm{Bi}$ and $\mathrm{Sb}$ compositions. The chemical composition of $\left(\mathrm{Bi}_{1-\mathrm{x}} \mathrm{Sb}_{\mathrm{x}}\right)_{2} \mathrm{Te}_{3}$ remains almost unchanged after the desorption of Te. This study proves that the Te capping layer works as a suitable protection for ternary $\left(\mathrm{Bi}_{1-\mathrm{x}} \mathrm{Sb}_{\mathrm{x}}\right)_{2} \mathrm{Te}_{3} \mathrm{TI}$ layers, allowing for ex-situ transfer of TI samples in air. This opens the way for the development of metal/TI hybrid structures for advanced spintronic applications.
\end{abstract}

Keywords: topological insulator; surface state bands; Dirac point; angle resolved photoemission spectroscopy; capping and desorption 


\section{Introduction}

Discovery of the new quantum state of matter called topological insulators (TI) has attracted world-wide interest due to their exotic properties which are manifestations of a non-trivial band topology $[1,2]$. TIs have insulating bulk and conducting edges due to the presence of peculiar surface state bands (SSBs). These SSBs are spin non-degenerated with a unique property of spin momentum locking which is resulted from the strong spin-orbit coupling (SOC) effects in combination with the time reversal symmetry $[3,4]$. Recently, it has been reported that the band structure of TI can be engineered by fabricating alloys of $\mathrm{Bi}_{2} \mathrm{Te}_{3}$ and $\mathrm{Sb}_{2} \mathrm{Te}_{3}$ using molecular beam epitaxy (MBE) to form the corresponding ternary $\left(\mathrm{Bi}_{1-\mathrm{x}} \mathrm{Sb}_{\mathrm{x}}\right)_{2} \mathrm{Te}_{3}$ alloy $[5,6]$. The topological surface state (TSS) and the associated energy position of the Dirac point (DP) in the band gap can then be tuned systematically with different $\mathrm{Bi} / \mathrm{Sb}$ concentrations. The transport properties of ternary $\mathrm{TI}$ alloys are also consistent with that of a single spin-polarized Dirac cone. This allows to overcome the disadvantage of binary topological insulators $\mathrm{Bi}_{1-\mathrm{x}} \mathrm{Sb}_{\mathrm{x}}$ [7], $\mathrm{Bi}_{2} \mathrm{Se}_{3}$ or $\mathrm{Bi}_{2} \mathrm{Te}_{3}[8,9]$, which always show conductive bulk states due to the defect-induced charge carriers. Therefore, the tunable Dirac cone structure in the ternary $\left(\mathrm{Bi}_{1-\mathrm{x}} \mathrm{Sb}_{\mathrm{x}}\right)_{2} \mathrm{Te}_{3} \mathrm{TI}$ allows to develop topological $p$ - $n$ junction devices $[10,11]$.

Recently, the discovery of giant spin Hall angle at room temperature in ferromagnet/TI heterostructures has also attracted intensive interests due to the possible applications to advanced spintronic devices, such as spin-orbit-torque magneto-resistive random-access memories and spin-torque oscillators $[12,13,14,15]$. Experimentally, to fabricate metal/TI heterostructures, it is required to either (1) build sophisticated integrated multi-deposition systems to grow in-situ the TI and other materials (metals, oxides, semiconductors) without breaking the vacuum, or (2) deposit a suitable capping layer on TI before the transfer through air and then remove the capping layer in another UHV chamber. As reported, the protection by the Se capping layer is not efficient because of the replacement of Te by Se up to $7 \mathrm{~nm}$ deep region beneath the surface, which strongly changes the surface chemical composition and modifies the surface state after desorption [16]. Some works 
have reported the use of $\mathrm{Te}$ for the capping layer on binary $\mathrm{Bi}_{2} \mathrm{Te}_{3}[17,18]$, but the influence of $\mathrm{Te}$ on the stoichiometry and on the SSB of ternary $\left(\mathrm{Bi}_{1-\mathrm{x}} \mathrm{Sb}_{\mathrm{x}}\right)_{2} \mathrm{Te}_{3}$ after desorption is still unclear.

In this study, we have performed a systematic study of the surface morphology, crystallinity, chemical composition as well as the band structure of $\left(\mathrm{Bi}_{1-\mathrm{x}} \mathrm{Sb}_{\mathrm{x}}\right)_{2} \mathrm{Te}_{3}$ after the desorption of the Te capping layer. Our results confirm a good recovery of SSBs of the $\left(\mathrm{Bi}_{1-\mathrm{x}} \mathrm{Sb}_{\mathrm{x}}\right)_{2} \mathrm{Te}_{3}$ ternary topological insulators with different $\mathrm{Bi}$ and $\mathrm{Sb}$ compositions. We have found that the chemical composition of $\left(\mathrm{Bi}_{1-\mathrm{x}} \mathrm{Sb}_{\mathrm{x}}\right)_{2} \mathrm{Te}_{3}$ remains almost unchanged after the desorption of Te. This study proves that the Te capping layer works as a suitable protection for ternary $\left(\mathrm{Bi}_{1-\mathrm{x}} \mathrm{Sb}_{\mathrm{x}}\right)_{2} \mathrm{Te}_{3} \mathrm{TI}$ layers, allowing for ex-situ transfer of TI samples in air.

\section{Experimental methods}

The $\left(\mathrm{Bi}_{1-\mathrm{x}} \mathrm{Sb}_{\mathrm{x}}\right)_{2} \mathrm{Te}_{3}$ ternary topological insulators were grown by co-evaporation method in a MBE system with a base pressure of $5 \times 10^{-10}$ torr. Firstly, the dielectric $\mathrm{SrTiO}_{3}(111)$ substrates were annealed at $930^{\circ} \mathrm{C}$ in oxygen atmosphere for 3 hours. Ten quintuple layers (QLs) of $\left(\mathrm{Bi}_{1-\mathrm{x}} \mathrm{Sb}_{\mathrm{x}}\right)_{2} \mathrm{Te}_{3}$ layers were then grown at $250^{\circ} \mathrm{C}$ followed by an annealing procedure at $280^{\circ} \mathrm{C}$ for half an hour. The growth rate was calibrated by a real-time reflection high-energy electron diffraction (RHEED) intensity oscillation. Supplementary Fig. S1 shows the RHEED pattern taken on one sample of 10 QL thick $\left(\mathrm{Bi}_{0.19} \mathrm{Sb}_{0.81}\right)_{2} \mathrm{Te}_{3}$. The sharpness of the feature provides a clear evidence for the high quality of the sample. Two different chemical composition of $\left(\mathrm{Bi}_{0.19} \mathrm{Sb}_{0.81}\right)_{2} \mathrm{Te}_{3}$ and $\left(\mathrm{Bi}_{0.75} \mathrm{Sb}_{0.25}\right)_{2} \mathrm{Te}_{3}$ were prepared in this study. The films were capped with Te layer at room temperature before being exposed to air. The RHEED pattern after deposition of Te capping layer (Supplementary Fig. S2) completely loses all diffraction lines and indicates an amorphous nature of the Te layer. The details of growth condition can be found elsewhere [5].

The sample was then transferred to another ultra-high vacuum (UHV) multi-chamber interconnected system, where we firstly performed the desorption of Te layer. The desorption procedure was controlled by X- 
ray photoemission spectroscopy (XPS). The sample surface crystallinity was characterized by RHEED and low-energy electron diffraction (LEED). The sample morphology was characterized by scanning electron microscopy (SEM). Scanning Auger microscopy (SAM) and Auger electron spectroscopy (AES) were used to examine the chemical distribution at the surface. Finally, angle resolved photoemission spectroscopy (ARPES) was employed to study the band structure.

\section{Results and discussion}

Control of Te desorption procedure with XPS

The Te desorption process was controlled by XPS measurements. XPS spectra have been obtained using a monochromatized Al-Ka X-rays source $(1486.6 \mathrm{eV})$ with an energy resolution of $250 \mathrm{meV}$. Our previous experiments show that the stoichiometry of the sample was changed in an irremediable manner when it was heated up to $240^{\circ} \mathrm{C}$. To avoid damaging the sample structure, we have carefully heated the sample to $200^{\circ} \mathrm{C}$ with a ramp of $10^{\circ} \mathrm{C} / \mathrm{min}$. XPS spectra were recorded each one hour after cooling down the sample from $200^{\circ} \mathrm{C}$ to the room temperature. Since the STO substrate is insulating, a large charging effect produces a shift of about $40 \mathrm{eV}$ in the XPS spectra observed. To remove this charging effect, we have deposited $10 \mathrm{~nm}$ of $\mathrm{Au}$ at the sample edge when the Te desorption procedure was completed. As identified in Fig. 1(c), the Fermi level of the sample revealed from the rising of density of states of valence band (VB) is exactly located at zero binding energy (BE), indicating no charging effect after deposition of Au. Fig. 1(a) shows the large-scale XPS spectrum of the $\left(\mathrm{Bi}_{0.19} \mathrm{Sb}_{0.81}\right)_{2} \mathrm{Te}_{3}$ sample after desorption of Te. Besides of $\mathrm{Te}, \mathrm{Sb}$ and $\mathrm{Bi}$ signals, peaks related to $\mathrm{C}, \mathrm{Mo}$, $\mathrm{O}$ and $\mathrm{Au}$ elements can also be observed. These extra signatures originate from the sample holder and from the edge of the sample recovered by $\mathrm{Au}$ due to the large illumination area of X-rays source and the large entrance of the analyzer.

Fig. 2(a) shows Te 3d spectra before and after desorption. Before Te desorption, small shoulders appear 
at $\mathrm{BE}$ of $575 \mathrm{eV}$ and $585 \mathrm{eV}$ beside the metallic contributions of $\mathrm{Te} 3 \mathrm{~d}_{5 / 2}$ and $3 \mathrm{~d}_{3 / 2}$. These additional signatures are assigned to the corresponding oxidized contributions of Te $3 \mathrm{~d}$, indicating the existence of a very thin TeOx layer on top of the surface due to the sample transfer through air. After Te desorption, these shoulders disappear, indicating the complete removal of $\mathrm{TeO}_{\mathrm{x}}$ layer. Fig. 2 (b) presents the intensity evolution of $\mathrm{Te}^{3} \mathrm{~d}_{5 / 2}, \mathrm{Bi} 4 \mathrm{f}_{7 / 2}$ and $\mathrm{Sb} 3 \mathrm{~d}_{3 / 2}$ core levels as a function of the annealing time. During Te desorption, the intensity of Te $3 \mathrm{~d}_{5 / 2}$ almost stays constant, while the intensities of $\mathrm{Bi}_{4} \mathrm{f}_{7 / 2}$ and $\mathrm{Sb} 3 \mathrm{~d}_{3 / 2}$ increase proportionally to the annealing duration until saturation after $3 \mathrm{~h}$. The intensity change of $\mathrm{Bi}$ and $\mathrm{Sb}$ before and after Te desorption allows us to estimate the thickness of the Te capping layer according to the formula: $I=I_{0} \exp ^{-t / \lambda}[19]$, where $I$ and $I_{0}$ are the intensity of peak before and after Te desorption, respectively. $t$ is the thickness of Te capping layer and $\lambda$ is the inelastic mean free path (IMFP) at the BE of elemental peak [20]. By using the $\mathrm{Bi} 4 \mathrm{f}_{7 / 2}$ core level $(\lambda \approx 2 \mathrm{~nm})$ for the estimation, we can estimate the thickness of the Te capping layer to be about $3 \mathrm{~nm}$.

Fig. 2(c-e) show the Te 3d, Bi 4f and $\mathrm{Sb} 3 \mathrm{~d}$ core level spectra for the two samples with different chemical compositions (( $\left.\mathrm{Bi}_{0.19} \mathrm{Sb}_{0.81}\right)_{2} \mathrm{Te}_{3}$ and $\left.\left(\mathrm{Bi}_{0.75} \mathrm{Sb}_{0.25}\right)_{2} \mathrm{Te}_{3}\right)$ after desorption. Both $\mathrm{Te} 3 \mathrm{~d}$ and $\mathrm{Bi} 4 \mathrm{f}$ core levels exhibit no shoulder, indicating that there is no more surface oxidation layer. However, small peaks around $529 \mathrm{eV}$ and $533 \mathrm{eV}$ can be still distinguished beside the metallic contribution of $\mathrm{Sb} 3 \mathrm{~d}_{5 / 2}$. Since no similar peaks are observed beside the contribution of $\mathrm{Sb} 3 \mathrm{~d}_{3 / 2}$, we can then exclude the possibility from the oxidation of $\mathrm{Sb}$. Therefore, these small peaks could be attributed to the $\mathrm{O}_{1 \mathrm{~s}}$ core level resulting from the contamination on the sample holder which is also probed by X-rays due to the large illumination area, as mentioned before. By comparison of the two samples, the tendency of the intensity change in Bi $4 \mathrm{f}$ and $\mathrm{Sb} 3 \mathrm{~d}$ peaks agrees with the composition of samples. However, the intensity change is still much smaller than what we expect from the nominal growth concentration. In fact, we expect a 4 and 3.5 times change in $\mathrm{Bi}$ and $\mathrm{Sb}$ signals, respectively. However, the change of $\mathrm{Bi} 4 \mathrm{f}$ and $\mathrm{Sb} 3 \mathrm{~d}$ intensities are only 1.3 and 2 times, respectively. In order to determine the surface stoichiometry, we have acquired spectra of $\mathrm{Te} 4 \mathrm{~d}$, Sb $4 \mathrm{~d}$ and $\mathrm{Bi} 5 \mathrm{~d}$ of the $\left(\mathrm{Bi}_{0.19} \mathrm{Sb}_{0.81}\right)_{2} \mathrm{Te}_{3}$ sample, 
as shown in Fig. 1(b). Since these peaks are located in a narrow BE range of $20-40 \mathrm{eV}$, we can avoid the different IMFP for different elements. After subtraction of the background, the Te $4 \mathrm{~d}, \mathrm{Sb} 4 \mathrm{~d}$ and $\mathrm{Bi} 5 \mathrm{~d}$ spectra have been fitted to extract the integrated area under the peaks. By taking into account the atomic sensitivity factor: Te 4d: $0.97, \mathrm{Sb} 4 \mathrm{~d}:$ 0.86, Bi $5 \mathrm{~d}: 0.65$ (from Ref. [21]), we obtain the stoichiometry to be about $\mathrm{Bi}: \mathrm{Sb}: \mathrm{Te}=1.5: 0.8: 3$. This composition is different than the nominal composition of $\mathrm{Bi}: \mathrm{Sb}: \mathrm{Te}=0.4: 1.6: 3$ determined by the flux ratio $\mathrm{Sb} / \mathrm{Bi}$ during the growth [5]. Since XPS results are more surface sensitive, our results indicate that the surface stoichiometry of TI could be different than the bulk one.

\section{Study of surface crystallinity, morphology and chemical composition after Te desorption}

RHEED and LEED have been used to characterize the surface crystallinity after desorption. In the inset of Fig. 3(a), the RHEED pattern is taken with an electron energy of $15 \mathrm{keV}$ at grazing incidence, i.e. parallel to the sample surface. Before Te desorption, RHEED pattern shows a featureless diffusive pattern due to the amorphous character of Te capping layer (Supplementary Fig. S1(b)). After Te desorption, the sharpness of the feature is compatible to the pattern before Te deposition (Supplementary Fig. S1(a)), which proves the high quality of the sample surface. The inset of Fig. 3(b) displays the LEED pattern taken with an electron energy of $59 \mathrm{eV}$ at normal incidence, i.e. perpendicular to the sample surface. In addition to the six-fold symmetric spots related to the diffraction of the epitaxially grown (111) plane of $\left(\mathrm{Bi}_{0.19} \mathrm{Sb}_{0.81}\right)_{2} \mathrm{Te}_{3}$, a ring associated to a polycrystalline structure is also observed. The different information given by RHEED and LEED could be related to their specific geometry and energy of electrons (coherence length) for characterization, which allows us to get complementary information on the surface crystallinity of the sample. Generally, LEED is more reliable for the analysis of defect structure due to the low inelastic scattering with low incident electron energy [22].

To understand the origin of the polycrystalline ring, the surface morphology is examined by SEM. Fig. 
3(a) and (b) show the SEM images with different scales. In the large-scale image in Fig.3(a), many flake-like island structures with size of several microns can be observed on the relative flat surface. These islands have random shape but all exhibit 2D growth mode with flat surface. In the magnified image in Fig. 3(b), some protrusion structures with small size of $50 \mathrm{~nm}$ can be distinguished. These protrusion structures are homogeneously distributed on the whole surface. To check if the flake-like islands and the protrusion features are related to the procedure of Te desorption, we have measured the surface morphology of one pristine TI surface before Te deposition with atomic force microscopy (AFM). The sample was scanned by AFM immediately after being taken out from UHV growth chamber. In Supplementary Fig. S2(b), similar flake-like feature can be distinguished with a much smaller island size (about 100nm) which could be due to the different chemical concentration of the observed sample. In the large-scale AFM image (Supplementary Fig. S2(a)), the protrusion structures with the same density and size can be found in the pristine surface. These results confirm that the specific features observed after Te desorption already exist on the TI pristine surface and the Te desorption procedure does not modify the surface morphology. The islands could be related to the 2D growth mode of $\left(\mathrm{Bi}_{0.19} \mathrm{Sb}_{0.81}\right)_{2} \mathrm{Te}_{3}$, which are firstly grown laterally and coalesce with each other to form the full film. Harrison et al. have reported the growth of $\mathrm{Bi}_{2} \mathrm{Te}_{3}$ thin films on $\mathrm{Al}_{2} \mathrm{O}_{3}(0001)$ substrate by MBE [23]. Similar protrusion $3 \mathrm{D}$ defect structures have been found on the surface of $\mathrm{Bi}_{2} \mathrm{Te}_{3}$, which are attributed to the limited temperature window for $\mathrm{Bi}_{2} \mathrm{Te}_{3}$ thin film growth. In our case, the protrusion structures could also be related to the $3 \mathrm{D}$ defect structure with small mis-orientated $\left(\mathrm{Bi}_{0.19} \mathrm{Sb}_{0.81}\right)_{2} \mathrm{Te}_{3}$ grains. However, at present stage, we cannot conclude that the polycrystalline ring found in LEED pattern is due to the protrusion 3D defects or the flakelike islands. To definitively answer this question, further spatial resolved surface structure analysis should be performed.

To characterize the distribution of surface chemical composition, we have performed scanning Auger microscopy (SAM) with a spatial resolution of $27 \mathrm{~nm}$. Fig. 3(e) shows the typical AES spectra for energy 
ranges of $88-109 \mathrm{eV}$ and $420-500 \mathrm{eV}$ on the island part, where we can distinguish the peaks of $\mathrm{Bi}, \mathrm{Sb}$ and $\mathrm{Te}$ located at kinetic energy (KE) of $97 \mathrm{eV}, 450 \mathrm{eV}$ and $479 \mathrm{eV}$, respectively. To explore the chemical distribution at the surface, we have chosen one zone including the island structure, as shown in the SEM image in Fig. 3(c). Since $\mathrm{Sb}$ and $\mathrm{Te}$ are very suitable for comparison due to the very closed KE and related IMFP, we have performed the mappings of $\mathrm{Sb}$ and Te elements, as shown in Fig. 3(d) and (f), respectively. In both images, we can observe a clear intensity contrast which well corresponds to the morphology of the surface in the vicinity of the island. The origin of this contrast is found to be related to the topography of the surface but not to its chemical composition. This has been checked by estimating the difference of intensity ratio of $I_{\mathrm{Sb}} /\left(I_{\mathrm{Te}}+I_{\mathrm{Sb}}\right)$ inside and outside the island, which is found to be less than $2 \%$. Furthermore, we also found the same intensity ratio in the protrusion structure (see Supplementary Fig. S3). Therefore, we can conclude that the chemical composition is homogeneous on the $\left(\mathrm{Bi}_{0.19} \mathrm{Sb}_{0.81}\right)_{2} \mathrm{Te}_{3}$ surface after Te desorption.

\section{Band structure characterization with ARPES}

We have studied the band structure of two samples with different concentrations ( $x=0.81$ and $x=0.25)$ by means of ARPES. ARPES measurements have been performed at a low photon energy using Xe-I radiation ( $h v=8.44 \mathrm{eV}$ ) for the $x=0.81$ sample and using He-I radiation $(h v=21.22 \mathrm{eV})$ for the $x=0.25$ one with an overall energy resolution including UV sources and analyzer characteristics better than $10 \mathrm{meV}$. The ARPES signal is expected to be more bulk sensitive by using Xe-I than He-I. However, we found that a higher signal to noise ratio was obtained with the Xe-I UV lamp compared to the He-I one. We also measured constant energy maps by using the deflection mode of our Scienta-omicron DA30 L photoemission analyzer, without rotating the sample. Here, the $k_{\mathrm{x}}$ axis corresponds to the standard measurement direction obtained along the analyzer slit, whereas the $k_{\mathrm{y}}$ axis corresponds to the perpendicular direction by using the deflectors. 
Fig. 4(c) shows the ARPES spectrum obtained on the $\left(\mathrm{Bi}_{0.19} \mathrm{Sb}_{0.81}\right)_{2} \mathrm{Te}_{3}$ sample after Te desorption. We can clearly observe the existence of the TSS within the gap of bulk states. The bulk valence band (BVB) is visible but not the bulk conduction band (BCB) associated with the $n$-type impurities. The TSS has a linear dispersion with two branches intersecting at DP at $\Gamma$ point (i.e. at $k_{/ /}=0$ ). These two branches have an opposite spin texture. The BE of the DP is $E_{\mathrm{D}}=-150 \pm 20 \mathrm{meV}$. The Fermi wave vectors $k_{\mathrm{F}}$ have been measured to be $\pm k_{\mathrm{F}}=0.06 \AA^{-1}$. The Fermi velocity $V_{\mathrm{F}}$ of the linear dispersion of the Dirac cone following $E-E_{\mathrm{F}}=\hbar V_{\mathrm{F}}\left(k-k_{\mathrm{F}}\right)$ is equal to $2.46 \pm 0.05 \times 10^{6} \mathrm{~m} / \mathrm{s}$. To compare with the band structure of the pristine TI sample without Te capping, we have adopted the measured band structures for $\left(\mathrm{Bi}_{1-x} \mathrm{Sb}_{x}\right)_{2} \mathrm{Te}_{3}(x=0.88$ and 0.75$)$ published from the same group [5], as shown in Fig. 4(a) and (b). Since the energy position of DP $\left(E_{\mathrm{D}}\right)$ is very sensitive to the stoichiometry of TI sample, this allows us to examine if the stoichiometry is changed after Te desorption. Interestingly, our results highlight that the DP position at $E_{\mathrm{D}}=-0.15 \mathrm{eV}$ for $\left(\mathrm{Bi}_{0.19} \mathrm{Sb}_{0.81}\right)_{2} \mathrm{Te}_{3}$ after Te desorption is well seated intermediately between the DP positions of $\left(\mathrm{Bi}_{0.12} \mathrm{Sb}_{0.88}\right)_{2} \mathrm{Te}_{3}\left(E_{\mathrm{D}}=-0.12 \mathrm{eV}\right)$ and $\left(\mathrm{Bi}_{0.25} \mathrm{Sb}_{0.75}\right)_{2} \mathrm{Te}_{3}$ $\left(E_{\mathrm{D}}=-0.21 \mathrm{eV}\right)$, which is in perfect agreement with Ref. [5]. Therefore, we can conclude a high quality of TI surface despite the process of Te capping and desorption. Furthermore, our results prove the high performance of Xe lamp, especially its high photon flux to discern the TSS band with very low spectral weight and located in the vicinity of the $\Gamma$ point. Fig. 4(d) and (e) present the constant energy maps at the Fermi energy $\left(E_{\mathrm{F}}\right)$ and at the DP energy position $\left(E_{\mathrm{D}}\right)$, respectively. In Fig. 4(d), the Fermi surface of the TI alloy displays an almost circular shape (black dashed circle), which is in agreement with an alloy with small concentration of $\mathrm{Bi}$, unlike the case of $\mathrm{Bi}_{2} \mathrm{Te}_{3}$ [24]. The circle diameter is shrunk for the energy close to DP (Fig. 4(e)), which validates the cone structure of TSS band.

The second TI sample $\left(\mathrm{Bi}_{0.25} \mathrm{Sb}_{0.75}\right)_{2} \mathrm{Te}_{3}$ with different $\mathrm{Bi} / \mathrm{Sb}$ ratio has also been characterized with ARPES after the same procedure of desorption of Te capping layer. The band structure has been recorded with a He I lamp source $(h v=21.2 \mathrm{eV})$, as shown in Fig. 5(b). Again, we can clearly observe the TSS dispersion in the gap 
of the bulk states. For this sample, the signature of BCB can be observed at the Fermi level inside the Dirac cone. The BVB is very similar to what has been observed in the case of a pure Bi(111) sample [25]. The BE of the DP is $E_{\mathrm{D}}=-250 \pm 20 \mathrm{meV}$. The Fermi wave vectors have been measured to be $\pm k_{\mathrm{F}}=0.12 \AA^{-1}$, close to the characteristic of compound $\mathrm{Bi}_{2} \mathrm{Te}_{3}$ [24]. The Fermi velocity of the linear dispersion of the Dirac cone following $E-E_{\mathrm{F}}=\hbar V_{\mathrm{F}}\left(k-k_{\mathrm{F}}\right)$ is equal to $1.00 \pm 0.05 \times 10^{7} \mathrm{~m} / \mathrm{s}$. We have also compared our results with the published result measured with the pristine TI sample with the same concentration [5] (Fig. 5(a)). The two results possess almost the same features, which validates the fact that the TSS is unchanged after Te desorption. In Fig. 5(c), we have plotted a series of momentum distribution curves (MDC) at $E=-45 \mathrm{meV}$ (close to $E_{\mathrm{F}}$ ), $-115 \mathrm{meV}$ and $230 \mathrm{meV}$ (close to $E_{\mathrm{D}}$ ). It allows us to better disentangle the band structure of the system. On one hand, MDC at $E=-45 \mathrm{meV}$ and $-115 \mathrm{meV}$ can be well fitted with three components which are related to the TSS and BCB dispersions. On the other hand, only one peak centered at $k_{\mathrm{x}}=0$ is found at $E=E_{\mathrm{D}}$. It corresponds to the crossing point of the two TSS branches at the $\Gamma$ point.

Fig. 5(d-g) display the constant energy maps recorded at different energies. Since the stoichiometry of the second TI sample is different from that of the first sample we studied, a change in the topology of the Fermi surface is expected. This is indeed what we observe in Fig. 5(e) at $E=E_{\mathrm{F}}-40 \mathrm{meV}$. We can see the electron pocket at the center of the Brillouin zone $\left(k_{\mathrm{x}}=k_{\mathrm{y}}=0\right)$ corresponding to the $\mathrm{BCB}$, as well as the deformation of the Fermi surface associated to the TSS. The singular topology "snow flake" of the Fermi surface of the spinpolarized TSS (marked with black dot line) can be well understood like in the case of $\mathrm{Bi}_{2} \mathrm{Te}_{3}$ material [24], which is also in good agreement with the data of the literature [26]. At $E=E_{\mathrm{F}}-100 \mathrm{meV}$ (Fig.4(f)), the isoenergetic surface regains a uniform circle character. Finally, at lower BE ( $\left.E=E_{\mathrm{F}}-250 \mathrm{meV}\right)(\mathrm{Fig} .4(\mathrm{~g}))$, the isoenergetic surface of the valence band is characteristic of a Bi(111) sample with this six-branched "flower" form [25], confirming the $\mathrm{Bi}$ rich composition in the measured $\left(\mathrm{Bi}_{0.75} \mathrm{Sb}_{0.25}\right)_{2} \mathrm{Te}_{3}$ sample. 


\section{Summary}

We have performed a systematic study of the surface morphology, crystallinity, chemical composition as well as the band structure of $\left(\mathrm{Bi}_{1-\mathrm{x}} \mathrm{Sb}_{\mathrm{x}}\right)_{2} \mathrm{Te}_{3}$ after the desorption of Te capping layer. Our results confirm a good recovery of $\mathrm{SSBs}$ of the $\left(\mathrm{Bi}_{1-\mathrm{x}} \mathrm{Sb}_{\mathrm{x}}\right)_{2} \mathrm{Te}_{3}$ ternary topological insulators with different $\mathrm{Bi}$ and $\mathrm{Sb}$ compositions. The chemical composition of $\left(\mathrm{Bi}_{1-\mathrm{x}} \mathrm{Sb}_{\mathrm{x}}\right)_{2} \mathrm{Te}_{3}$ remains almost unchanged after the desorption of Te. This study proves that the Te capping layer works as a suitable protection for ternary TI layers, allowing for ex-situ transfer of TI samples in air. This opens the way for the development of metal/TI hybrid structures for advanced spintronic applications.

\section{Acknowledgement}

Y.Lu acknowledges the support from the French National Research Agency (ANR) FEOrgSpin project (Grants No. ANR-18-CE24-0017-01) and ICEEL international SHATIPN project. K. Zhu and K. He acknowledge the support from the National Key Research and Development Program of China under Grant No 2017YFA0303303. Experiments were performed using equipment from the platform TUBE-DAUM funded by FEDER (EU), ANR, the Region Lorraine and Grand Nancy. 


\section{Figures}

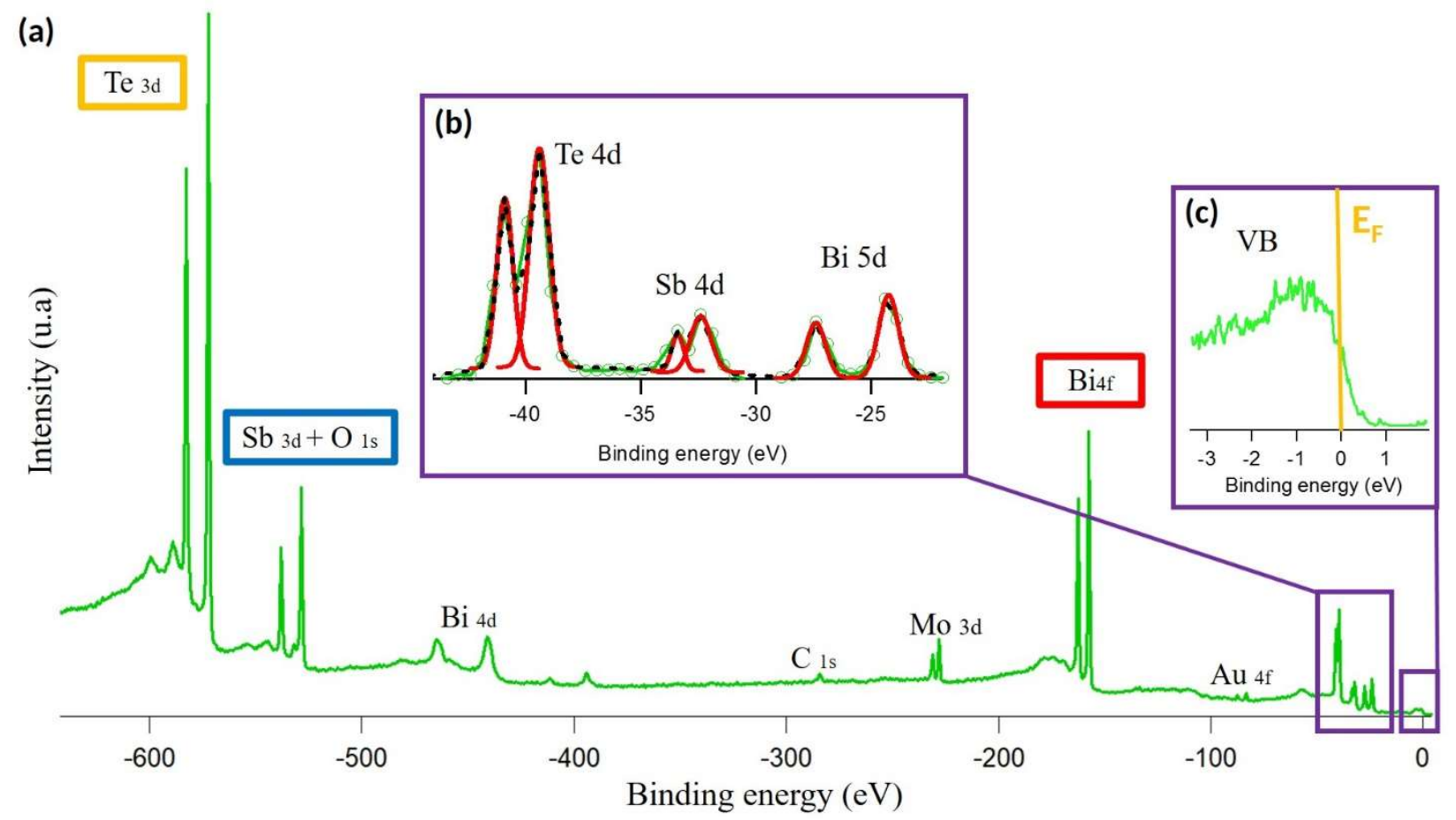

Figure 1: (a) General XPS spectrum obtained with monochromatized Al-K $\alpha$ radiation $(1486.6 \mathrm{eV})$ on the $\left(\mathrm{Bi}_{0.19} \mathrm{Sb}_{0.81}\right)_{2} \mathrm{Te}_{3}$ sample. (b) Zoom on the low energy part to show $\mathrm{Te} 4 \mathrm{~d}, \mathrm{Sb} 4 \mathrm{~d}$ and $\mathrm{Bi} 5 \mathrm{~d}$ core levels (after subtracting a Shirley background). The red curves indicate the peak fitting allowing to quantify the stoichiometry. (c) Zoom at the top of the valence band close to the Fermi level. 

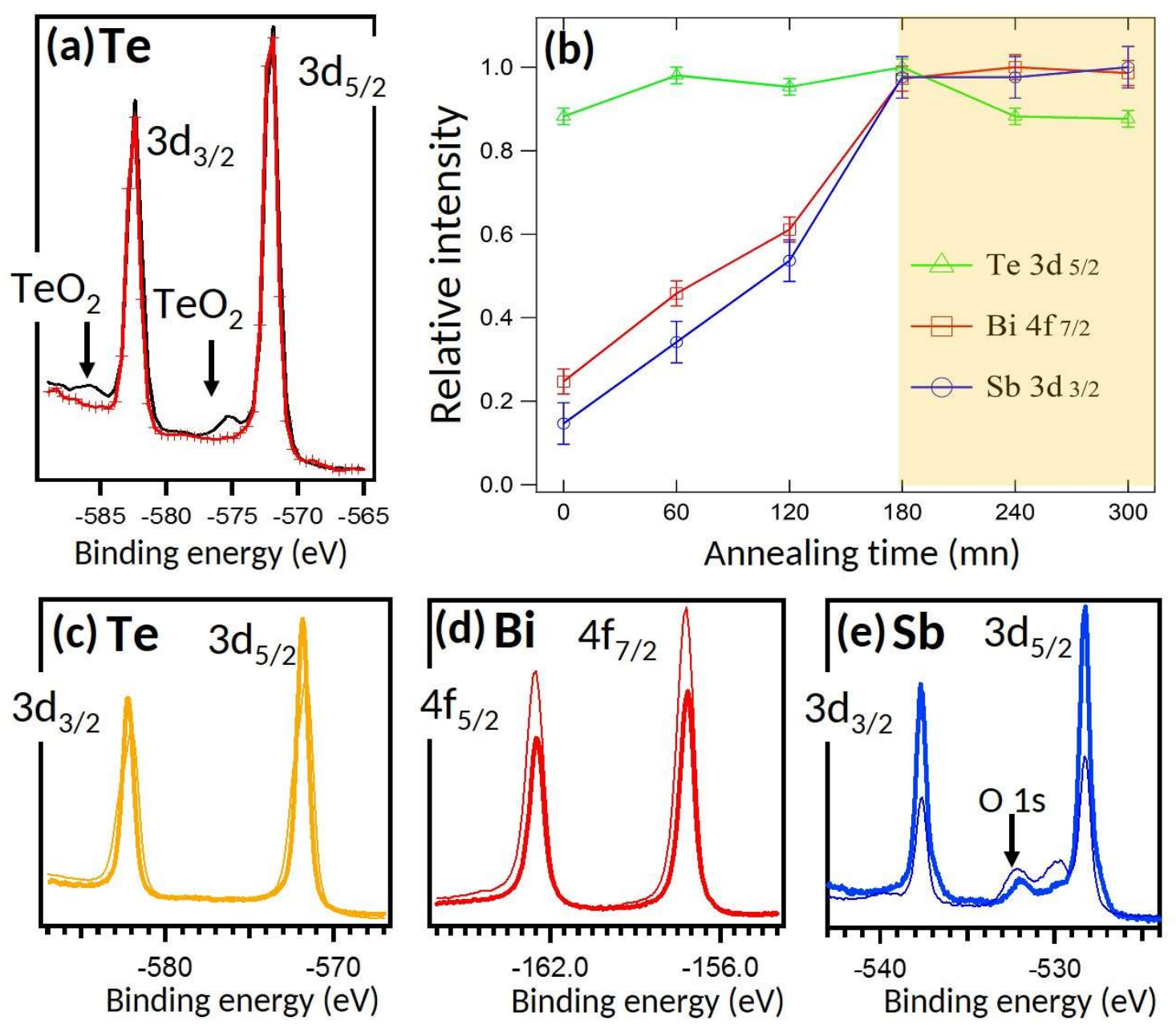

Figure 2: (a) Zoom on the Te 3d core level before the desorption (black curve) and after the desorption (red curve). (b) Evolution of Bi 4f, Sb 3d and Te 3d core level intensity with the annealing time. (c-e) XPS core levels for (c) Te 3d, (d) Bi 4f and (e) Sb 3d after Te desorption for $\left(\mathrm{Bi}_{1-x} \mathrm{Sb}_{x}\right)_{2} \mathrm{Te}_{3}$ sample 1 ( $x=0.81$, bold lines) and sample $2(x=0.25$, thin lines). 


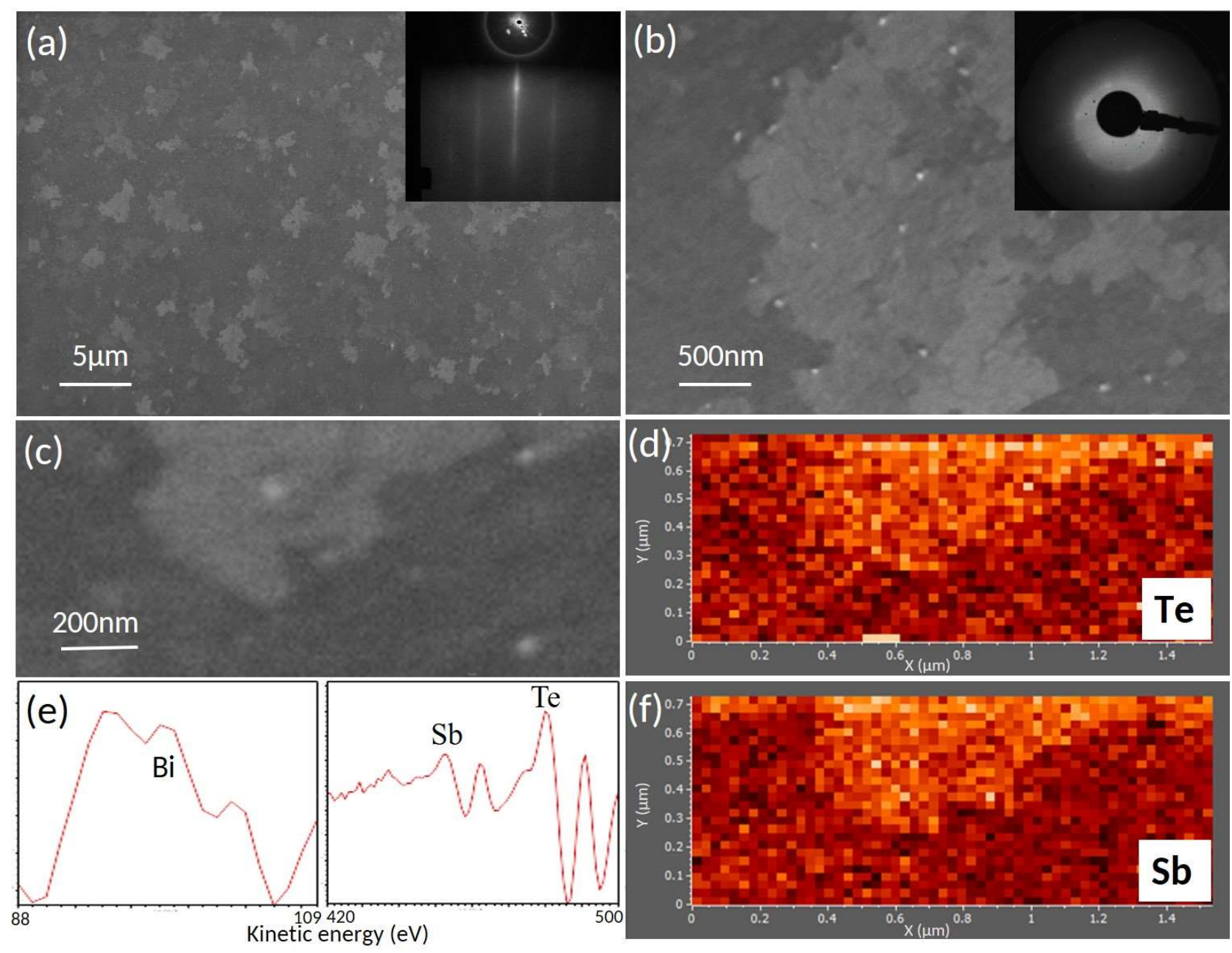

Figure 3: $(\mathrm{a}, \mathrm{b}) \mathrm{SEM}$ images with different scales for $\left(\mathrm{Bi}_{0.19} \mathrm{Sb}_{0.81}\right)_{2} \mathrm{Te}_{3}$ sample after desorption. Inset of (a):

RHEED pattern of the surface after Te desorption. Inset of (b): LEED pattern of surface after desorption. (c)

SEM image revealing an island feature on the surface. $(\mathrm{d}, \mathrm{f})$ SAM mapping taken in the area of panel (c) for

(d) Te and (f) Sb signals. (e) Typical AES spectra for Bi, $\mathrm{Sb}$ and Te signals. 

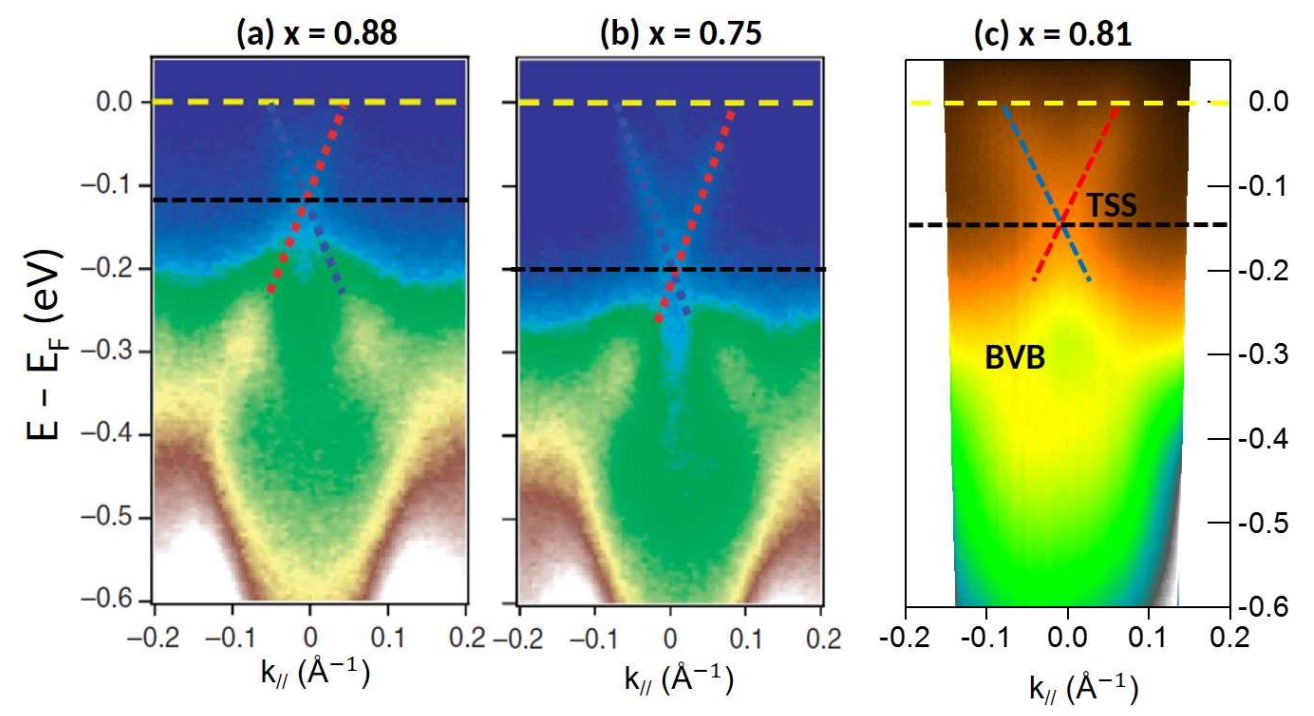

(d) $\mathrm{E}=\mathrm{E}_{\mathrm{F}}$

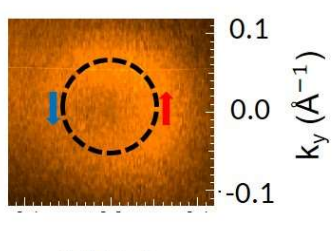

(e) $E=E_{D}$

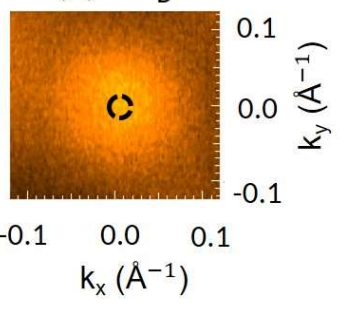

Figure $4:(a, b)$ ARPES intensity maps on pristine $\left(\mathrm{Bi}_{1-\mathrm{x}} \mathrm{Sb}_{\mathrm{x}}\right)_{2} \mathrm{Te}_{3}$ surfaces without Te capping obtained from

Ref. [5] for the concentration of (a) $x=0.88$ and (b) $x=0.75$. (c) ARPES intensity map in this work using Xe-I photons of $8.44 \mathrm{eV}$ for $\left(\mathrm{Bi}_{0.19} \mathrm{Sb}_{0.81}\right)_{2} \mathrm{Te}_{3}$ surface after Te desorption. The topological surface state (TSS) and the bulk valence band (BVB) are clearly evidenced. (d,e) ARPES constant energy maps obtained at (d) $E=E_{\mathrm{F}}$ and (e) $E=E_{\mathrm{D}}=E_{\mathrm{F}}-150 \mathrm{meV}$. Black dotted lines are guide to the eyes. Blue and red arrows indicate the in-plane spin polarization. 
(a) $x=0.25$

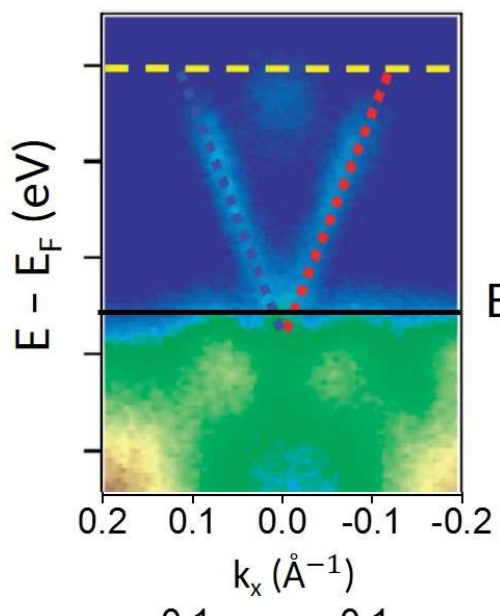

0.1

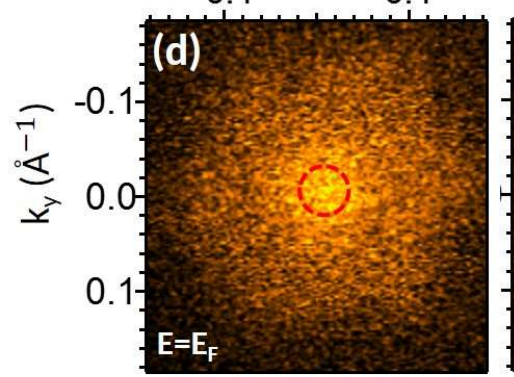

$k_{x}\left(\AA^{-1}\right)$ (b) $x=0.25$
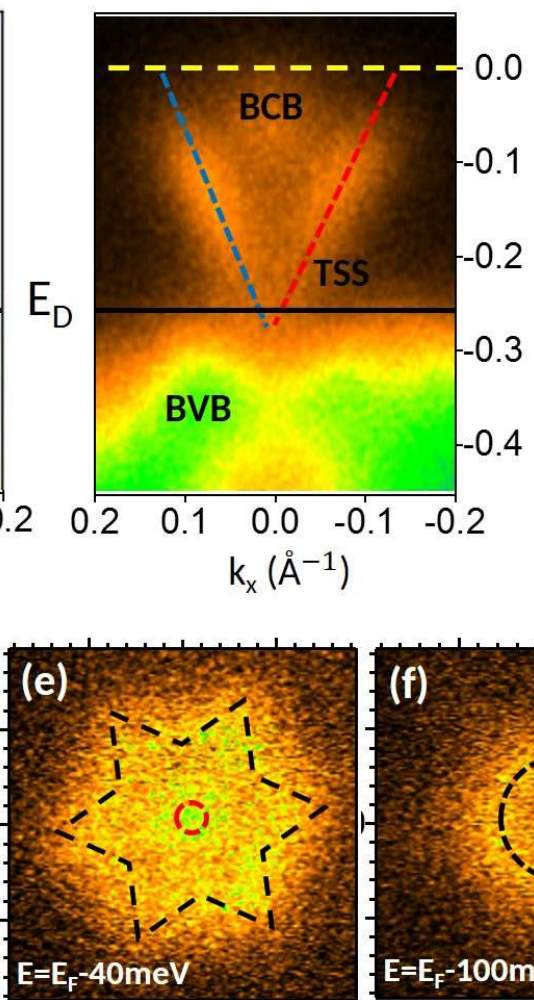

$\mathrm{k}_{\mathrm{x}}\left(\AA^{-1}\right)$

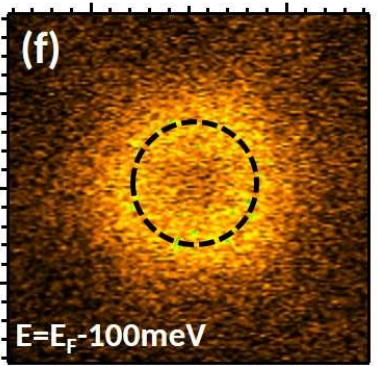

$k_{x}\left(\AA^{-1}\right)$ (c) MDCs
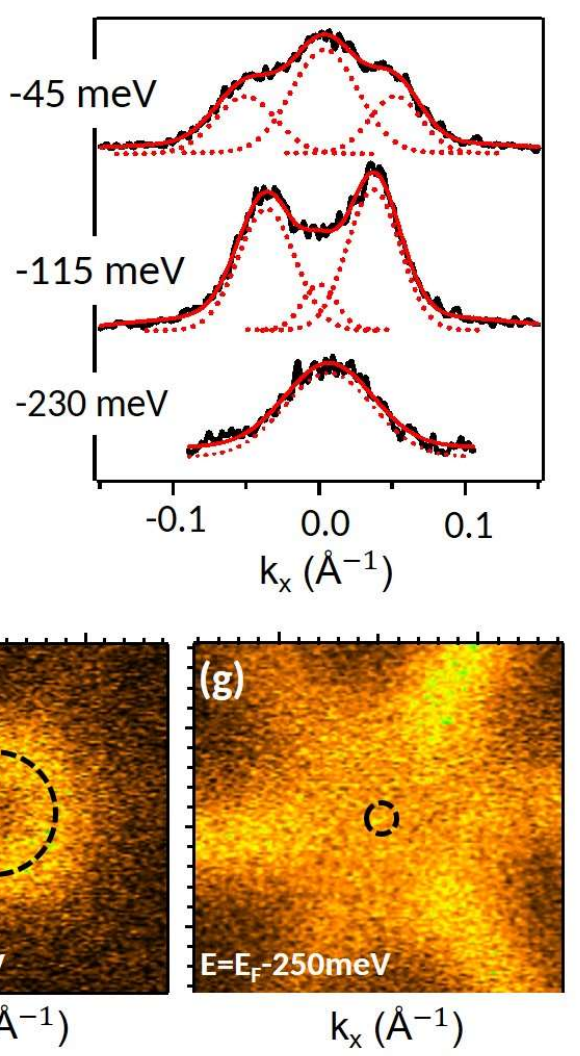

Figure 5 : (a) ARPES intensity maps on pristine $\left(\mathrm{Bi}_{1-\mathrm{x}} \mathrm{Sb}_{\mathrm{x}}\right)_{2} \mathrm{Te}_{3}$ surfaces without Te capping obtained from

Ref. [5] for the concentration of $x=0.25$. (b) ARPES intensity map in this work using He-I photons of 21.22

Ev for $\left(\mathrm{Bi}_{0.75} \mathrm{Sb}_{0.25}\right)_{2} \mathrm{Te}_{3}$ surface after Te desorption. The bulk conduction band $(\mathrm{BCB})$, the TSS and the BVB are clearly evidenced. (c) Corresponding momentum dispersion curves (MDCs) at three typical energies. (dg) ARPES constant energy maps obtained at (d) $E=E_{\mathrm{F}}$, (e) $E=E_{\mathrm{F}}-40 \mathrm{meV}$, (f) $E=E_{\mathrm{F}}-100 \mathrm{meV}$ and (g) $E=E_{\mathrm{D}}=E_{\mathrm{F}}-$ $250 \mathrm{meV}$. Red and black dotted lines are guide to the eyes. 


\section{References:}

[1] X. L. Qi and S. C. Zhang, “Topological insulators and superconductors”, Rev. Mod. Phys. 83, 1057 (2011).

[2] M. G. Vergniory, L. Elcoro, C. Felser, N. Regnault, B. A. Bernevig and Z. J. Wang, “A complete catalogue of high-quality topological materials", Nature 566, 480-485 (2019).

[3] R. Pedram, J. Seo, C. V. Parker, Y. S. Hor, D.Hsieh, D.Qian, A. Richardella, M. Z. Hasan, R. J. Cava, and A. Yazdani, "Topological surface states protected from backscattering by chiral spin texture", Nature 460, 1106 (2009).

[4] T. Zhang, P. Cheng, X. Chen, J.-F. Jia, X. Ma, K. He, L. Wang, H. Zhang, X. Dai, Z. Fang, X. Xie, and Q.-K. Xue, "Experimental Demonstration of Topological Surface States Protected by Time-Reversal Symmetry", Phys. Rev. Lett. 103, 266803 (2009).

[5] J. Zhang, C. Z. Chang, Z. Zhang, J. Wen, X. Feng, K. Li, M. Liu, K. He, L. Wang, X. Chen, Q.-K. Xue, X. $\mathrm{Ma}$ and $\mathrm{Y}$. Wang, "Band structure engineering in $\left(\mathrm{Bi}_{1-x} \mathrm{Sb}_{x}\right)_{2} \mathrm{Te}_{3}$ ternary topological insulators", Nature Communications 2, 574 (2011).

[6] C. Weyrich, M. Drögeler, J. Kampmeier, M. Eschbach, G. Mussler, T. Merzenich, T. Stoica, I. E. Batov, J. Schubert, L. Plucinski, "Growth, characterization, and transport properties of ternary $\left(\mathrm{Bi}_{1-x} \mathrm{Sb}_{x}\right)_{2} \mathrm{Te}_{3}$ topological insulator layers", J. Phys.: Condens. Matter 28, 495501 (2016).

[7] D. Hsieh, D. Qian, L. Wray, Y. Xia, Y. S. Hor, R. J. Cava and M. Z. Hasan, “A topological Dirac insulator in a quantum spin Hall phase", Nature 452, 970-974, (2008).

[8] D. Hsieh, Y. Xia, D. Qian, L. Wray, F. Meier, J. H. Dil, J. Osterwalder, L. Patthey, A. V. Fedorov, H. Lin, A. Bansil, D. Grauer, Y. S. Hor, R. J. Cava, and M. Z. Hasan, "Observation of Time-Reversal-Protected SingleDirac-Cone Topological-Insulator States in $\mathrm{Bi}_{2} \mathrm{Te}_{3}$ and $\mathrm{Sb}_{2} \mathrm{Te}_{3}$ ”, Phys. Rev. Lett. 103, 146401 (2009).

[9] Y. Xia, D. Qian, D. Hsieh, L. Wray, A. Pal, H. Lin, A. Bansil, D. Grauer, Y. S. Hor, R. J. Cava and M. Z. Hasan, "Observation of a large-gap topological-insulator class with a single Dirac cone on the surface", Nature Physics 5, 398-402 (2009).

[10] S. H. Kim, K.-H. Jin, B. W. Kho, B.-G. Park, F. Liu, J. S. Kim and H. W. Yeom, “Atomically Abrupt Topological p-n Junction", ACS Nano 11, 9671-9677 (2017).

[11] N. H. Tu, Y. Tanabe, Y. Satake, K. K. Huynh and K. Tanigaki, "In-plane topological p-n junction in the three-dimensional topological insulator $\mathrm{Bi}_{2-\mathrm{x}} \mathrm{Sb}_{\mathrm{x}} \mathrm{Te}_{3-\mathrm{y}} \mathrm{Se}_{\mathrm{y}}$ ", Nature Communications 7, 13763 (2016).

[ 12 ] Y. Fan, P. Upadhyaya, X. Kou, M. Lang, S. Takei, Z. Wang, J. Tang, L. He, L.-T. Chang, M. Montazeri, G. Yu, W. Jiang, T. Nie, R. N. Schwartz, Y. Tserkovnyak and K. L. Wang, "Magnetization switching through giant spin-orbit torque in a magnetically doped topological insulator heterostructure", Nature Materials 13, 699-704 (2014).

[13] K. Kondou, R. Yoshimi, A. Tsukazaki, Y. Fukuma, J. Matsuno, K. S. Takahashi, M. Kawasaki, Y. Tokura and Y. Otani, "Fermi-level-dependent charge-to-spin current conversion by Dirac surface states of topological insulators", Nature Physics 12, 1027-1031 (2016).

[14] N. H. D. Khang, Y. Ueda and P. N. Hai, "A conductive topological insulator with large spin Hall effect for ultralow power spin-orbit torque switching", Nature Materials 17, 808-813 (2018). 
[15] M. DC, R. Grassi, J.-Y. Chen, M. Jamali, D. R. Hickey, D. Zhang, Z. Zhao, H. Li, P. Quarterman, Y. Lv, M. Li, A. Manchon, K. A. Mkhoyan, T. Low and J.-P. Wang, "Room-temperature high spin-orbit torque due to quantum confinement in sputtered $\mathrm{Bi}_{x} \mathrm{Se}_{(1-\mathrm{x})}$ films", Nature Materials 17, 800-807 (2018).

[16] K. Virwani, S. E. Harrison, A. Pushp, T. Topuria, E. Delenia, P. Rice, A. Kellock, L. C.-McIntyre , J. Harris, T. Hesjedal , and S. Parkin, "Controlled removal of amorphous Se capping layer from a topological insulator”, Appl. Phys. Lett. 105, 241605 (2014).

[17] K. Hoefer, C. Becker, S. Wirth, and L. H. Tjeng "Protective capping of topological surface states of intrinsically insulating $\mathrm{Bi}_{2} \mathrm{Te}_{3}$ ”, AIP Advances 5, 097139 (2015).

[18] C. I. Fornari, P. H. O. Rappl, S. L. Morelhão, T. R. F. Peixoto, H. Bentmann, F. Reinert, and E. Abramof, "Preservation of pristine $\mathrm{Bi}_{2} \mathrm{Te}_{3}$ thin film topological insulator surface after ex situ mechanical removal of Te capping layer", APL Materials 4, 106107 (2016).

[19] K. Hirokawa, S. Suzuki, K. Abiko, H. Kimura and M. Oku, "Estimation of the thickness or composition of a covering layer on a solid by XPS or AES", Journal of Electron Spectroscopy and Related Phenomena 24, 243-253 (1981).

[20] M. P. Seah and W. A. Dench, "Quantitative Electron Spectroscopy of Surfaces: A Standard Data Base for Electron Inelastic Mean Free Paths in Solids", Surface and Interface Aanalysis 1, 2 (1979).

[21] C. D. Wagner, L. E. Davis, M. V. Zeller, J. A. Taylor, R. M. Raymond and L. H. Gale, "Empirical atomic sensitivity factors for quantitative analysis by electron spectroscopy for chemical analysis", Surf. Interface Anal. 3, 211 (1981).

[22] B. Mfiller, M. Henzler, "Comparison of reflection high-energy electron diffraction and low energy electron diffraction using high-resolution instrumentation”, Surface Science 389, 338-348 (1997).

[23] S. E. Harrison, S. Li, Y. Huo, B. Zhou, Y. L. Chen, and J. S. Harris, "Two-step growth of high quality $\mathrm{Bi}_{2} \mathrm{Te}_{3}$ thin films on $\mathrm{Al}_{2} \mathrm{O}_{3}$ (0001) by molecular beam epitaxy", Appl. Phys. Lett. 102, 171906 (2013).

[24] S. Souma, K. Kosaka, T. Sato, M. Komatsu, A. Takayama, T. Takahashi, M. Kriener, K. Segawa, and Y. Ando, "Direct measurement of the out-of-plane spin texture in the dirac-cone surface state of a topological insulator," Phys. Rev. Lett. 106, 21 (2011).

[25] Y. Ohtsubo, L. Perfetti, M. O. Goerbig, P. L. Fèvre, F. Bertran and A. Taleb-Ibrahimi, "Non-trivial surface-band dispersion on Bi(111)", New Journal of Physics 15, 033041 ( 2013).

[26] M. Nomura, S. Souma, A. Takayama, T. Sato, T. Takahashi, K. Eto, Kouji Segawa, and Yoichi Ando, "Relationship between Fermi surface warping and out-of-plane spin polarization in topological insulators: A view from spin- and angle-resolved photoemission", Phys. Rev. B 89, 045134 (2014). 